\title{
Overweight and Obesity are Risk Factors for Coronavirus Disease 2019: A Propensity Score-Matched Case-Control Study
}

\author{
Wonjun $\mathrm{Ji}^{1}{ }^{1 *}$, Rugyeom Lee ${ }^{2, *}$, Kyungmin $\mathrm{Huh}^{3}$, Minsun $\mathrm{Kang}^{2}$, In Cheol Hwang ${ }^{4}$, Munkhzul Radnaabaatar ${ }^{2}$, \\ Dae Ho Lee ${ }^{5}$ Jaehun Jung ${ }^{2,6}$
}

${ }^{1}$ Department of Pulmonary and Critical Care Medicine, Asan Medical Center, University of Ulsan College of Medicine, Seoul; ${ }^{2}$ Artificial Intelligence and Big-Data Convergence Center, Gachon University Gil Medical Center, Gachon University College of Medicine, Incheon; ${ }^{3}$ Division of Infectious Diseases, Department of Medicine, Samsung Medical Center, Sungkyunkwan University School of Medicine, Seoul; Departments of ${ }^{4}$ Family Medicine, ${ }^{5}$ Internal Medicine, Gachon University Gil Medical Center, Gachon University College of Medicine; ${ }^{6}$ Department of Preventive Medicine, Gachon University College of Medicine, Incheon, Korea

Although obesity is a risk factor for infection, whether it has the same effect on coronavirus disease 2019 (COVID-19) need confirming. We conducted a retrospective propensity score matched case-control study to examine the association between obesity and COVID-19. This study included data from the Nationwide COVID-19 Registry and the Biennial Health Checkup database, until May 30, 2020. We identified 2,231 patients with confirmed COVID-19 and 10-fold-matched negative test controls. Overweight (body mass index [BMI] 23 to $24.9 \mathrm{~kg} / \mathrm{m}^{2}$; adjusted odds ratio [aOR], 1.16; 95\% confidence interval [CI], 1.1.03 to 1.30) and class 1 obesity (BMI 25 to $29.9 \mathrm{~kg} / \mathrm{m}^{2}$; aOR, 1.27; 95\% CI, 1.14 to 1.42) had significantly increased COVID-19 risk, while classes 2 and 3 obesity (BMI $\geq 30 \mathrm{~kg} / \mathrm{m}^{2}$ ) showed similar but non-significant trend. Females and those $<50$ years had more robust association pattern. Overweight and obesity are possible risk factors of COVID-19.

Keywords: COVID-19; Obesity; Overweight; Infections

\section{INTRODUCTION}

Coronavirus disease 2019 (COVID-19), caused by the novel se-

Received: 23 September 2020, Revised: 14 December 2020,

Accepted: 19 January 2021

Corresponding authors: Jaehun Jung

Department of Preventive Medicine, Gachon University College of Medicine, 38 Dokjeom-ro 3beon-gil, Namdong-gu, Incheon 21565, Korea

Tel: +82-32-458-2611, Fax: +82-32-458-2608, E-mail: eastside1st@gmail.com

Dae Ho Lee

Department of Internal Medicine, Gachon University Gil Medical Center, Gachon University College of Medicine, 21 Namdong-daero 774beon-gil, Namdong-gu, Incheon 21565, Korea

Tel: +82-32-458-2733, Fax: +82-32-468-5836, E-mail: drhormone@naver.com vere acute respiratory syndrome coronavirus 2 (SARS-CoV-2), has resulted in $>28$ million confirmed cases and 917,000 deaths as of mid-September 2020 [1]. Although previous epidemiolog-

\footnotetext{
Copyright $\odot 2021$ Korean Endocrine Society

This is an Open Access article distributed under the terms of the Creative Commons Attribution Non-Commercial License (https://creativecommons.org/ licenses/by-nc/4.0/) which permits unrestricted non-commercial use, distribution, and reproduction in any medium, provided the original work is properly cited.
}

*These authors contributed equally to this work. 
ical studies reported that obesity was associated with infectious diseases risk [2-4], whether it has the same effect on COVID-19 needs confirming.

We conducted a retrospective propensity score (PS) matched case-control study, to examine association between obesity and COVID-19 risk using data from the Nationwide Registry of COVID-19 cases and the Biennial Health Checkup database of South Korea.

\section{METHODS}

\section{Statement of ethics}

This research was conducted in accordance with the ethics requirements of the World Medical Association Declaration of Helsinki and approved by Institutional Review Board of the Gachon University College of Medicine, Incheon, Republic of Korea (GFIRB2020-118), and written informed consent requirement was waived, since human subjects were not involved in the study.

\section{Data sources}

Data were obtained from the Korea Center for Disease Control and Prevention (KCDC) Nationwide COVID-19 Registry and the Biennial Health Checkup database of the National Health Insurance Service (NHIS) of South Korea, until May 30, 2020. The NHIS database was linked to the KCDC COVID-19 registry.

The data regarding comorbidities (identified using International Statistical Classification of Diseases and Related Health Problems, 10th Revision) were extracted from the NHIS reimbursement database and categorized in Supplemental Table S1. The comorbidities, identified from diagnostic codes, included at least twice according to the reimbursement records covering the past 3 years and dated before COVID-19 onset.

\section{Study patients and definitions}

Patients aged $\geq 20$ years who underwent laboratory test for COVID-19 and NHIS health checkup in 2018 and later, were included. COVID-19 diagnosis was made using nasopharyngeal swab or sputum sample, and analyzed using reverse transcriptase polymerase chain reaction to detect SARS-CoV-2 according to the national guidelines [5]. All patients who tested positive were categorized as confirmed cases and the remaining as negative controls. Data on body mass index (BMI) were categorized as normal (BMI $<23 \mathrm{~kg} / \mathrm{m}^{2}$ ), overweight (BMI 23.0 to $24.9 \mathrm{~kg} / \mathrm{m}^{2}$ ), class 1 obesity (BMI 25.0 to $29.9 \mathrm{~kg} / \mathrm{m}^{2}$ ), and classes 2 and 3 obesity (BMI $\geq 30 \mathrm{~kg} / \mathrm{m}^{2}$ ) according to the Ko- rean Society for the Study of Obesity guidelines (Supplemental Table S2) [6]. As the primary outcome, association between infection of COVID-19 and BMI categories was analyzed, and association with fasting blood sugar (FBS) was also evaluated.

\section{Statistical analysis}

Baseline characteristics of COVID-19 patients and test negative controls were compared using chi-square test and Student's $t$ test, as appropriate. Charlson comorbidity index (CCI) was calculated using standard methods [7] and compared. Adjusted logistic regression models were constructed, based on the following covariates: age, sex, NHIS expanded medical aid coverage, and comorbidities. We constructed PS matched cohorts to mitigate the impact of confounders. PSs for COVID-19 risk were calculated using logistic regressions including the following covariates: age, sex, medical aid coverage, and CCI. Each patient with COVID-19 was matched with up to 10 test negative controls using the greedy neighbor nearest-matching algorithm (Fig. 1). The model was further adjusted in a logistic regression analysis, using age, sex, insurance medical aid coverage, and comorbidities, as covariates. The standardized mean difference in PS matching are presented in Supplemental Table S3. All statistical tests were two-tailed and the threshold for significance was set at $P$ values $<0.05$. All analyses were performed using SAS software version 9.4 (SAS Institute Inc., Cary, NC, USA).

\section{RESULTS}

\section{Baseline demographics between cases and test negative controls}

A total of 76,332 individuals aged $\geq 20$ years who underwent a laboratory test for COVID-19 and the NHIS health checkup in 2018 and later, were identified. From the 74,101 negative test controls, 10-fold PS matched controls were selected and analyzed (Fig. 1).

The proportion of females $(1,360$ of 2,$231 ; 61.0 \%)$ and those with medical aid (142 of 2,$231 ; 6.4 \%$ ) were higher among positive cases than the negative controls. COVID-19 patient group were older than those in the control group. After PS matching, the matched cohort showed similar baseline characteristics except for the proportion with medical aid, diabetes, chronic liver disease, malignancy, and rheumatologic disease (Supplemental Table S4).

\section{Association between BMI category and COVID-19}

Overall, higher BMI showed positive association with COV- 


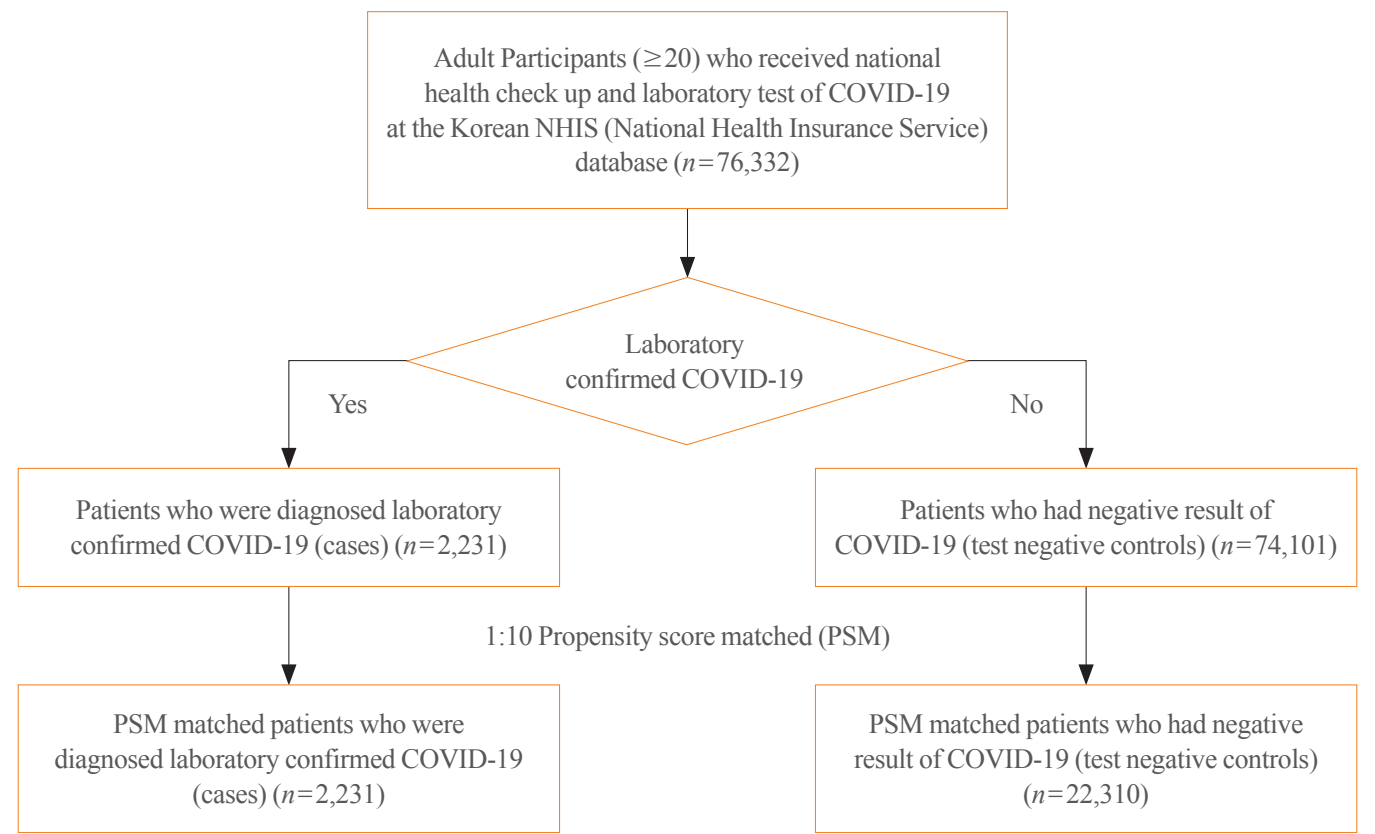

Fig. 1. Flowchart of the patient selection process. COVID-19, coronavirus disease 2019; NHIS, National Health Insurance Service.
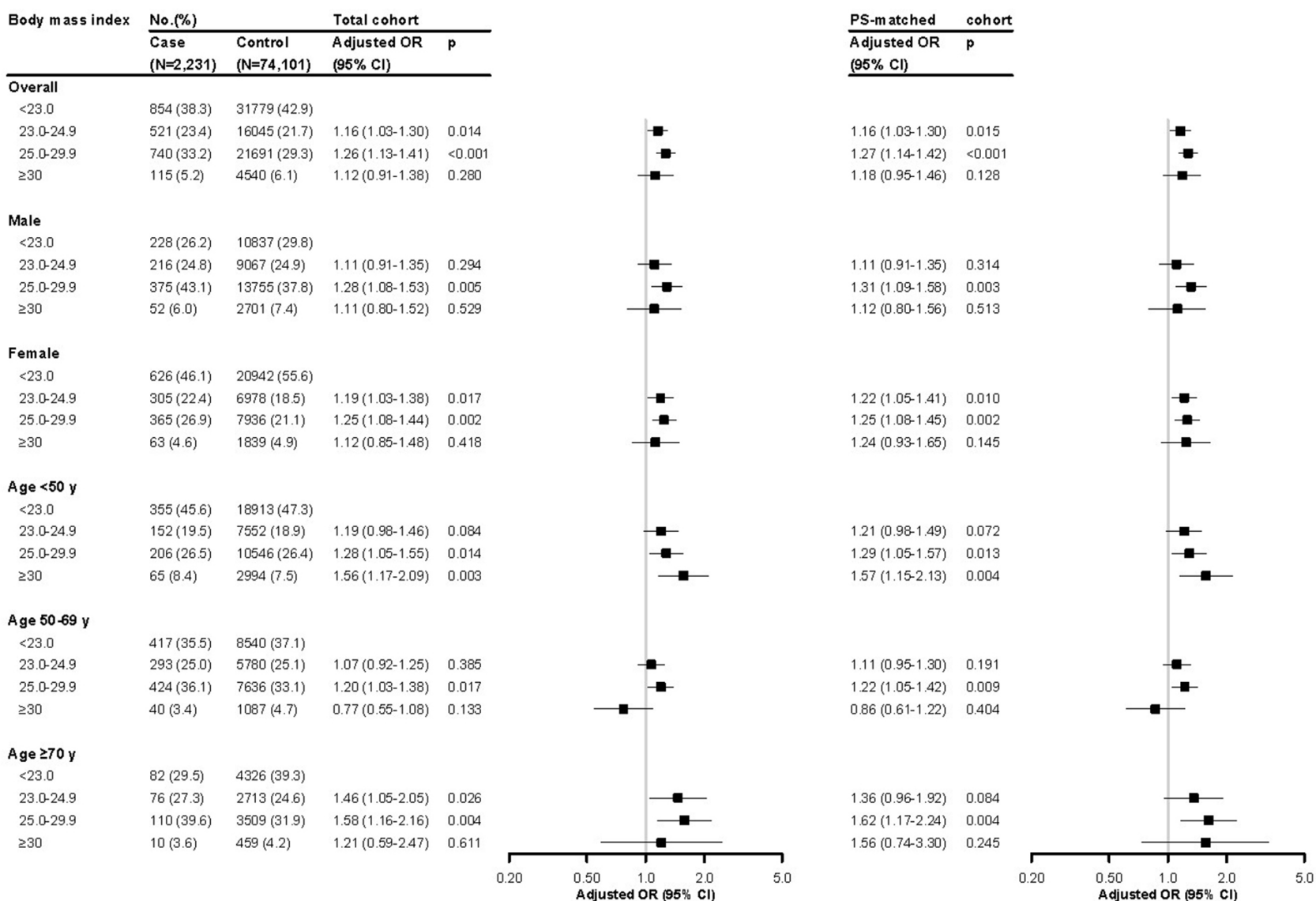

Fig. 2. Adjusted odds ratios (ORs) for patients who were confirmed with coronavirus disease 2019 and the negative test controls. CI, confidence interval; PS, propensity score. 
ID-19 (Fig. 2). Patients who were overweight (adjusted odds ratio [aOR], 1.16; 95\% confidence interval [CI], 1.03 to $1.30 ; P=$ 0.015 ) and had class 1 obesity (aOR, $1.27 ; 95 \% \mathrm{CI}, 1.14$ to 1.42 ; $P<0.001$ ) had significantly increased risk of COVID-19 compared to those with normal BMI reading, while patients with classes 2 and 3 obesity showed similar but not significant trend (aOR, 1.18; 95\% CI, 0.95 to $1.46 ; P=0.128$ ). The effect of BMI on the risk of COVID-19 was more prominent among women than among men. By age group, the association patterns of overweight and class 1 obesity were robust for all age groups. Classes 2 and 3 obesity showed a significant association in $<50$ years age group (aOR, 1.57; 95\% CI, 1.15 to $2.13 ; P=0.004$ ).

\section{Association between fasting blood glucose category and COVID-19}

FBS $\geq 126 \mathrm{mg} / \mathrm{dL}$ was significantly associated with COVID-19 only in females (aOR, 1.33; 95\% CI, 1.05 to $1.69 ; P=0.02$ ), while the other groups and overall analysis did not show significant differences (Supplemental Table S5).

\section{DISCUSSION}

In this study, we found overweight and obesity as possible risk factors of COVID-19, especially in females and in < 50 years age group. Our findings are consistent with previous reports, which revealed obesity as a significant risk factor for the occurrence, severity, and mortality of COVID-19 [8-12]. Increased expression levels of angiotensin-converting enzyme 2 by adipocytes, overactive renin-angiotensin system due to higher levels of angiotensin II, endothelial dysfunction, and higher risk of thromboembolism have been proposed as possible mechanisms linking obesity with COVID-19 risk [13-15].

The present study found that class 1 obesity (BMI 25.0 to $29.9 \mathrm{~kg} / \mathrm{m}^{2}$ ) was also a significant risk factor for COVID-19. Moreover, higher BMI and FBS $\geq 126 \mathrm{mg} / \mathrm{dL}$ were more significantly associated with COVID-19 in females than in males. Some previous studies reported that there may be gender differences in susceptibility to COVID-19 [16,17], and the present study results also suggest this. Interestingly, classes 2 and 3 obesity were significantly associated with increased COVID-19 risk in $<50$ years age group, while a similar but not significant trend was shown in the older age groups. A small retrospective study reported obesity as a predictive factor affecting COVID-19 severity in young adults $<40$ years old [18]. This previous report is supported by findings of our study indicating that obesity may have a greater impact on COVID-19 in younger adults.

This study has a few limitations. First, there is the possibility of selection bias. We used data of patients who underwent health checkups in 2018 or later, and it is possible that these patients were more likely to have undergone health checkups, as they may have had more comorbidities. However, to mitigate this confounding factor, we used a robust design with the PS matched analysis. Moreover, we consider that the results reflected more accurately the current body weight, because we used the most recent heath checkup data. Second, we were unable to evaluate the mechanism of the relationship between obesity and COVID-19. However, this study findings confirmed the association between obesity and COVID-19 and we recommend that detailed mechanisms of the relationship be evaluated by further experimental research.

In conclusion, overweight and obesity are possible risk factors of COVID-19, especially in females and in those aged $<50$ years. Therefore, weight and obesity approaches are important considerations in COVID-19 pandemic prevention and control.

\section{CONFLICTS OF INTEREST}

No potential conflict of interest relevant to this article was reported.

\section{ACKNOWLEDGMENTS}

The authors would like to thank all the healthcare professionals dedicated to the treatment of patients with COVID-19 in Korea; and the Ministry of Health and Welfare and the National Health Insurance Service of Korea for their prompt sharing of invaluable national health insurance claims data.

This study was supported by grants from the Gachon University Gil Medical Center (grant numbers 2019-11) and a grant from the Korea Health Technology Research and Development Project through the Korea Health Industry Development Institute, funded by the Ministry of Health and Welfare, Korea (grant numbers HI14C1135). The sponsors of the study were not involved in the study design, analysis, interpretation of data, and the writing of the report; nor the decision to submit the findings of the study for publication.

\section{AUTHOR CONTRIBUTIONS}

Conception or design: W.J., K.H., I.C.H., D.H.L., J.J. Acquisition, analysis, or interpretation of data: W.J., R.L., K.H., I.C.H., 
D.H.L., J.J. Drafting the work or revising: W.J., D.H.L. Final approval of the manuscript: W.J., R.L., K.H., M.K., I.C.H., M.R., D.H.L., J.J.

\section{ORCID}

Wonjun Ji https://orcid.org/0000-0001-7164-2770

Rugyeom Lee https://orcid.org/0000-0002-4507-4877

Dae Ho Lee https://orcid.org/0000-0002-8832-3052

Jaehun Jung https://orcid.org/0000-0002-4856-3668

\section{REFERENCES}

1. World Health Organization. Coronavirus disease (COVID-19) Weekly Epidemiological Update and Weekly Operational Update [Internet]. Geneva: WHO; 2020 [cited 2021 Jan 30]. Available from: https://www.who.int/emergencies/ diseases/novel-coronavirus-2019/situation-reports.

2. Dobner J, Kaser S. Body mass index and the risk of infection: from underweight to obesity. Clin Microbiol Infect 2018; 24:24-8.

3. Kaspersen KA, Pedersen OB, Petersen MS, Hjalgrim H, Rostgaard K, Moller BK, et al. Obesity and risk of infection: results from the Danish Blood Donor Study. Epidemiology 2015;26:580-9.

4. Maccioni L, Weber S, Elgizouli M, Stoehlker AS, Geist I, Peter $\mathrm{HH}$, et al. Obesity and risk of respiratory tract infections: results of an infection-diary based cohort study. BMC Public Health 2018;18:271.

5. Korea Center for Disease Control and Prevention. COVID-19 Response Guidelines (Edition 8-1) [Internet]. Sejong: KCDC; 2020 [cited 2021 Jan 30]. Available from: http:// ncov.mohw.go.kr/shBoardView.do?brdId $=2 \&$ brdGubu $n=2$ $8 \&$ ncvContSeq $=2447$.

6. Seo MH, Lee WY, Kim SS, Kang JH, Kang JH, Kim KK, et al. 2018 Korean Society for the Study of Obesity guideline for the management of obesity in Korea. J Obes Metab Syndr 2019;28:40-5.

7. Charlson ME, Pompei P, Ales KL, MacKenzie CR. A new method of classifying prognostic comorbidity in longitudinal studies: development and validation. J Chronic Dis 1987; 40:373-83.

8. Popkin BM, Du S, Green WD, Beck MA, Algaith T, Herbst $\mathrm{CH}$, et al. Individuals with obesity and COVID-19: a global perspective on the epidemiology and biological relationships. Obes Rev 2020;21:e13128.

9. Wang J, Zhu L, Liu L, Zhao XA, Zhang Z, Xue L, et al. Overweight and obesity are risk factors of severe illness in patients with COVID-19. Obesity (Silver Spring) 2020;28: 2049-55.

10. Singh S, Bilal M, Pakhchanian H, Raiker R, Kochhar GS, Thompson CC. Impact of obesity on outcomes of patients with coronavirus disease 2019 in the United States: a multicenter electronic health records network study. Gastroenterology 2020;159:2221-5.

11. Hamer M, Gale CR, Kivimaki M, Batty GD. Overweight, obesity, and risk of hospitalization for COVID-19: a community-based cohort study of adults in the United Kingdom. Proc Natl Acad Sci U S A 2020;117:21011-3.

12. Czernichow S, Beeker N, Rives-Lange C, Guerot E, Diehl JL, Katsahian S, et al. Obesity doubles mortality in patients hospitalized for severe acute respiratory syndrome coronavirus 2 in Paris hospitals, France: a cohort study on 5,795 patients. Obesity (Silver Spring) 2020;28:2282-9.

13. Sanchis-Gomar F, Lavie CJ, Mehra MR, Henry BM, Lippi G. Obesity and outcomes in COVID-19: when an epidemic and pandemic collide. Mayo Clin Proc 2020;95:1445-53.

14. Pasquarelli-do-Nascimento G, Braz-de-Melo HA, Faria SS, Santos IO, Kobinger GP, Magalhaes KG. Hypercoagulopathy and adipose tissue exacerbated inflammation may explain higher mortality in COVID-19 patients with obesity. Front Endocrinol (Lausanne) 2020;11:530.

15. Cinti S, Graciotti L, Giordano A, Valerio A, Nisoli E. COVID-19 and fat embolism: a hypothesis to explain the severe clinical outcome in people with obesity. Int J Obes (Lond) 2020;44:1800-2.

16. Lubrano C, Masi D, Risi R, Balena A, Watanabe M, Mariani $\mathrm{S}$, et al. Is growth hormone insufficiency the missing link between obesity, male gender, age, and COVID-19 severity? Obesity (Silver Spring) 2020;28:2038-9.

17. Rapp JL, Lieberman-Cribbin W, Tuminello S, Taioli E. Male sex, severe obesity, older age, and chronic kidney disease are associated with COVID-19 severity and mortality in New York City. Chest 2021;159:112-5.

18. Deng M, Qi Y, Deng L, Wang H, Xu Y, Li Z, et al. Obesity as a potential predictor of disease severity in young COVID-19 patients: a retrospective study. Obesity (Silver Spring) 2020;28:1815-25. 